\title{
Aberration retrieval for high-NA optical systems using the Extended Nijboer-Zernike theory
}

\author{
Peter Dirksen $^{a}$, Joseph J.M. Braat ${ }^{b}$, Augustus J.E.M. Janssen ${ }^{c}$, Ad Leeuwestein ${ }^{c}$ \\ ${ }^{a}$ Philips Research Leuven, Belgium \\ ${ }^{b}$ Delft University of Technology, The Netherlands \\ ${ }^{c}$ Philips Research Laboratories, The Netherlands
}

\begin{abstract}
Previously, we have given a detailed description of the so-called Extended Nijboer-Zernike approach and its application to aberration measurements of the optical projection system in a wafer scanner in the case of a low or medium high-NA system. The Extended Nijboer-Zernike theory provides an analytical description of the through-focus intensity point-spread function in the presence of lens aberrations and defocus.

Taking the Extended Nijboer-Zernike description for the electric field components in the case of a high-NA optical system as a starting point, we present an approach to aberration retrieval when the NA is very high. The experimental procedure involves the analysis of a focus-exposure matrix. The differences between aberration retrieval using the low-NA scalar model and the high-NA full vectorial model are discussed. The mathematical framework is shown and the experimental procedure to extract aberrations for a high-NA lens is demonstrated on modern $193 \mathrm{~nm}$ wafer scanners.
\end{abstract}

Keywords: Optical lithography, high NA, vectorial diffraction formalism, point-spread function, Extended Nijboer-Zernike theory.

\section{INTRODUCTION}

Optical lithography continues to increase its resolution, not only by reducing the exposure wavelength, but also by increasing the numerical aperture of the projection lens. A gradual increase through the years can be observed that partially explains the continuous increase in packing density on integrated circuits. At the same time, a few steps in wavelength have been made, from the near UV towards the actual deep UV at $193 \mathrm{~nm}$. The total increase in packing density over the years has only been possible by a drastic reduction in the value of the $k_{1}$-parameter. The projection lenses have contributed to this by a steady reduction of the aberrational level. Rms-values as low as $25 m \lambda$ are current nowadays. For this reason, a lithographic projection system has to be very carefully manufactured and monitored during its life with respect to aberrational level. Figure 1 shows a historical overview of the evolution of the numerical aperture. The fast increase starting in 2005 is caused by the introduction of immersion systems. Immersion lithography, with a fluid between the last lens element and the wafer, allows a numerical aperture greater than 1 . These so-called hyper-NA tools are scheduled within one year.

Important optical properties of an optical system can be obtained from the intensity point-spread function or impulse response. ${ }^{1}$ In optical lithography, an exact knowledge of the point-spread function is required to calculate the image of an object. Projection lens aberrations cause the point-spread function to deviate from its ideal shape that is well approximated by the Airy intensity distribution at moderate values of the numerical aperture. The impact of lens aberrations on the image performance of the lens is well known and described by various authors. ${ }^{2-5}$

Further author information: (Send correspondence to peter.dirksen@philips.com)

PD: Philips Research Leuven, Kapeldreef 75, B-3001 Leuven, Belgium

JB: Optics Research Group, Department of Applied Sciences, Delft University of Technology, Lorentzweg 1, NL-2628 CJ

Delft, The Netherlands

AJEMJ and AL: Philips Research Laboratories, NL-5656 AA Eindhoven, The Netherlands 


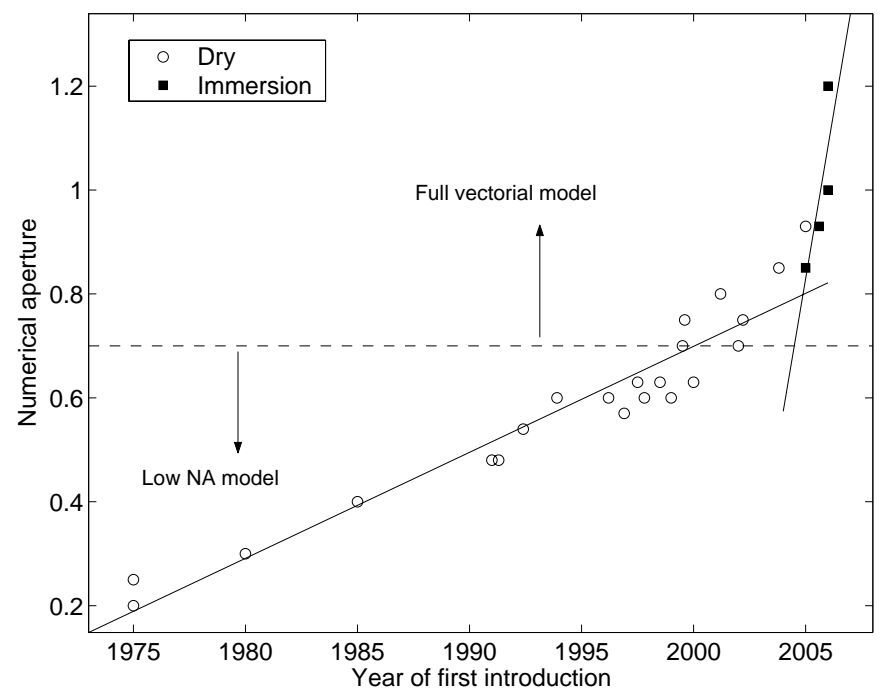

Figure 1. The evolution of the numerical aperture over the past 30 years. The sharp increase in 2005 is caused by the introduction of liquid immersion tools, allowing a numerical aperture in excess of 1. For small NA-values the scalar ENZ theory is sufficiently accurate, for NA-values above 0.7 the high-NA ENZ theory is needed.

The Extended Nijboer-Zernike (ENZ) theory provides an analytical description of the PSF. The throughfocus image intensity of the point-spread function, including the effects of aberrations and image blur caused by diffusion and focus noise, is described by a recently found Bessel series representation. ${ }^{9}$ These new series representation yield a flexible means to compute optical point-spread functions, both accurately and efficiently, under conditions that seem to cover almost all cases of practical interest. Due to the analytical nature of the formulas the ENZ theory is not only useful to do computations in the forward direction, i.e. to evaluate the point-spread function starting from aberrations, but also in the reverse direction, which amounts to aberration retrieval from the measured 3-D intensity point-spread function.

Up till now, the scalar ENZ theory was used for aberration retrieval and for estimating the DAIM parameters. ${ }^{6,7}$ However, it is well known that the point-spread function calculated by scalar theory deviates from the high-NA calculation when the NA exceeds a value of about 0.70. Aberration retrieval, therefore, becomes increasingly inaccurate when the NA is very high. ${ }^{20}$ Moreover, one tends to overestimate the diffusion parameter of the DAIM model as a scalar calculation tends to produce a PSF with a smaller lateral extent compared to the vectorial calculation. This paper discusses the extension to high-NA optical systems using the full vectorial ENZ theory in order to improve the accuracy of the retrieved parameters.

The paper is organized as follows. Section 1.1 gives an overview of ENZ publications in the period 2002 2005. Section 2 summarizes low-NA forward calculation and low-NA aberration retrieval. Section 3 summarizes the high-NA full vectorial forward calculations. In the special case of an unpolarized system with rotational symmetrically aberrations, we discusse aberrations retrieval and validate the high-NA formulas by comparing the results to SOLID-C simulation results. Section 4 shows some experimental results obtained on an ASML PAS 5500/1150 scanner and on an ASML Twinscan XT:1250i immersion scanner.

\subsection{Overview ENZ theory}

The Nijboer-Zernike aberration theory is described in the 1942-thesis of B.R.A. Nijboer. ${ }^{8}$ The Extended NijboerZernike theory was formulated for the first time ${ }^{9}$ in 2002 , where the basic diffraction integrals were represented in the form of easily computable power-Bessel series (Lommel type series). The forward calculation and an assessment of the validity of the new theory was given in a companion paper. ${ }^{10}$ Here it was shown that the through-focus point-spread function can be calculated for an optical system with arbitrary aberrations. The new Bessel series representation yields a flexible means to compute optical point-spread functions, both accurately 
and efficiently, under defocus and aberration conditions that seem to cover almost all cases of practical interest. Because of the analytic nature of the formulas, there are no discretization effects limiting the accuracy as opposed to the more commonly used numerical packages based on brute force integration methods. The old diffraction theories of Airy (1835), Lommel (1885) and Nijboer (1942) arise as special cases of the Extended Nijboer-Zernike theory.

The proof-of-principle of the backward calculation or retrieval of aberrations is described in Ref. ${ }^{11}$ The measurement method was demonstrated both analytically and experimentally. The extension to general aberration retrieval is described in Ref., ${ }^{13}$ where the retrieval of a general aberration function, $A \cdot \exp (i \Phi)$ with a possible non-constant pupil transmission amplitude $A$, was considered. In addition, the extension to finite pin-hole size is described. This makes the method much more practical as larger pinholes allow transmission of more light and thus significantly reduce the required exposure dose. In Ref. ${ }^{14}$ we show various experimental results performed on a modern wafer scanner.

Reference $^{15}$ describes the so-called extended diffused aerial image model (DAIM) and a method to retrieve its parameters. The DAIM parameters are at the origin of a general blurring of the projected aerial image and include the effects of diffusion and focus noise. The experimental procedure was demonstrated for several 193 $\mathrm{nm}$ resists under various conditions of post exposure baking temperature and baking time. Recent experimental results are discussed in Ref., ${ }^{12}$ on this conference.

A new calculation scheme to compute point-spread functions for any, small or large defocus value and at any spatial point is presented in Ref. ${ }^{16}$ The resulting Bessel-Bessel series expression, involving products of Bessel functions, can be shown to be practically immune to loss-of-digits.

The retrieval of large aberration values was discussed in Ref. ${ }^{17}$ By adopting an iterative approach, the effect of linearization can be eliminated iteratively for a surprisingly large range of aberration parameter values, and this yields accurate or even perfect retrieval of aberrations well beyond the diffraction limit. In addition, the impact of measurement noise on the retrieved aberrations was investigated.

The Extended Nijboer Zernike approach can also be applied to aberrated optical system with high NA by using the vectorial diffraction formalism. ${ }^{18}$ The relationship between the properties of the imaging system and the focal field distribution paves the way for a possible inverse problem solution, i.e. the retrieval of the complex pupil function from intensity measurements in the focal region for high-NA systems. In Refs. ${ }^{19,} 20$ it was shown that the basic methods employed in the previous scalar approach can still serve as a starting point for high-NA aberration retrieval. Using a vectorial analysis of aberrated imaging systems, a procedure to retrieve the complex pupil function of a high-NA imaging system is given.

Additional information can be found on the "Extended Nijboer-Zernike website". ${ }^{21}$

\section{THEORY: LOW NA - ENZ THEORY}

For low-to-moderate numerical aperture values of, say, NA $<0.70$, the scalar Extended Nijboer-Zernike approach applies. In this section we reproduce some formulas of the low-NA forward calculation. The electrical field is approximated by a scalar function:

$$
E \approx 2 V_{00}+2 i \sum_{n, m} \alpha_{n m} \cdot i^{m} V_{n m} \cos m \phi
$$

where $\alpha_{n m}$ are the Zernike coefficients corresponding to the single aberrations $R_{n}^{m}(\rho) \cos m \theta$. The definitions of the $V_{n m}$ functions can be found elsewhere ${ }^{9-11,13}$ and are not reproduced here. The intensity $I(r, \phi, f)$ is found as the square modulus of the field:

$$
I(r, \phi, f) \propto E \cdot E^{*} .
$$




\subsection{Low-NA aberration retrieval}

The next step is to apply a Fourier analysis with respect to the angular dependence of the observed image intensity:

$$
\Psi^{m}(r, f)=\frac{1}{2 \pi} \int_{0}^{2 \pi} I(r, \phi, f) \cos m \phi \mathrm{d} \phi .
$$

The physical interpretation of the $m^{t h}$ Fourier component is as follows: The Fourier component $\Psi^{0}(r, f)$, with $m=0$, includes the aberration-free and spherical aberration terms. For a high-quality lens the aberration-free term is dominant. The components $\Psi^{1}(r, f)$ and $\Psi^{2}(r, f)$ represent the contributions of coma and astigmatism, etc. Thus the Fourier analysis splits information according to aberration type. In addition, the Fourier analysis strongly suppresses the impact of measurement noise, making the retrieval method robust.

In order to retrieve the aberration coefficients, the $m^{\text {th }}$ harmonic of the observed intensity point-spread function $\Psi^{m}(r, f)$ is expressed as a linear sum of the $\Psi_{n}^{m}(r, f)$ basic intensity functions:

$$
\Psi^{m}(r, f) \approx \sum_{n} \alpha_{n m} \Psi_{n}^{m}(r, f) \text { with } \Psi_{n}^{m}(r, f) \propto \operatorname{Re}\left\{i^{m+1} V_{00}^{*} V_{n m}\right\} .
$$

In general, the aberration coefficients $\alpha_{n m}$ are found by a matching procedure between the observed and theoretical Fourier components. Referring to Figure 2, we demonstrate our algorithm that is valid for small aberration coefficients. A NA $=0.2$ microscope objective lens suffers from a mixture of several aberrations: spherical, astigmatism and trefoil aberration. Here we want to determine the dominant term: a small amount of low order trefoil aberration. We therefore extract the experimental third harmonic $\Psi^{3}(r, f)$ from the through-focus
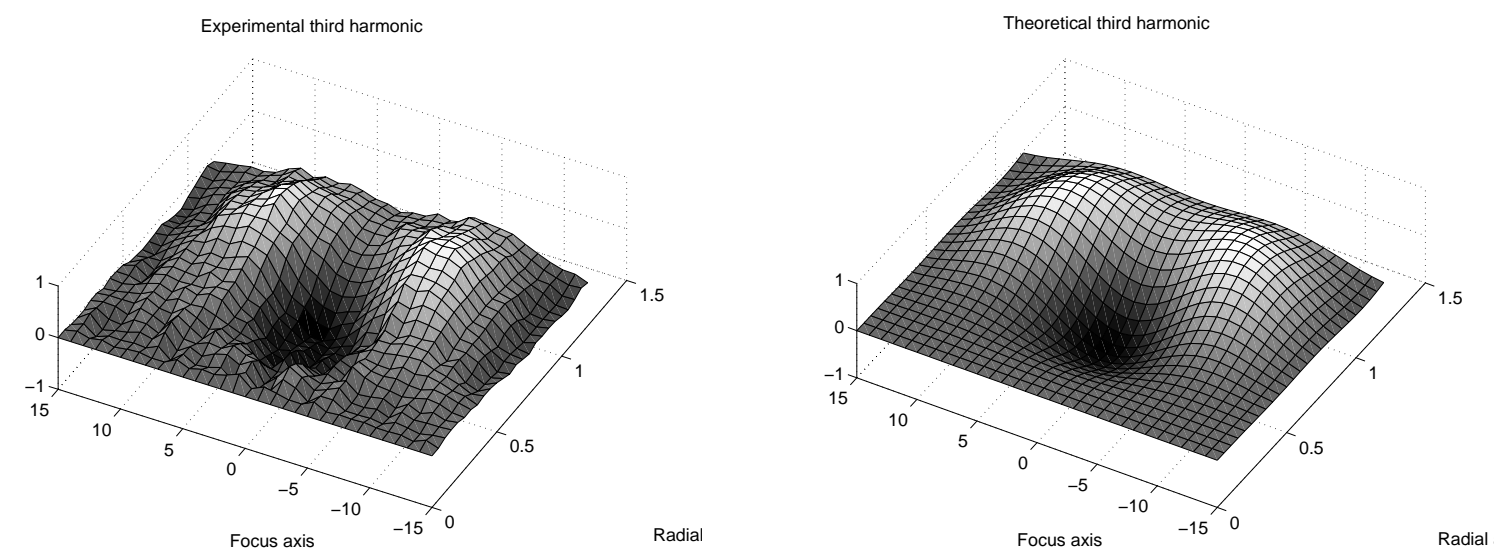

Figure 2. A comparison between the experimental third harmonic on the left and theoretical third harmonic on the right for a low NA microscope objective lens. The scaling factor $\alpha_{33}$ represents the amount of trefoil aberration.

intensity PSF, as shown in Figure 2 left, and match this experimental function to the theoretical $\Psi_{3}^{3}(r, f)$ basic intensity function shown in Figure 2 right:

$$
\Psi^{3}(r, f) \approx \alpha_{33} \Psi_{3}^{3}(r, f) .
$$

The proportionality coefficient $\alpha_{33}$ is found by a simple matching procedure. In general, also higher order aberrations may be present, and these can be found by solving a linear system of equations. This method can be extended to include transmission errors, effects of diffusion, focus noise and the finite diameter of the pinhole. For the retrieval of general, large aberrations an iterative scheme exists as discussed in the literature overview presented in the previous section.

\section{THEORY: HIGH-NA PSF CALCULATIONS}

For a numerical aperture that exceeds the value of 0.7 , the vectorial character of the light becomes important. Instead of the scalar expression in Eq. 1, the electrical field is now represented as a vector. The starting point 
of the high-NA aberration retrieval procedure is a set of high-NA papers ${ }^{18-20}$ on the high-NA extension of the ENZ theory. Assuming linearly polarized illumination along the x-axis, the electrical field in the image space is now given by:

$$
\mathbf{E}^{x}(r, \phi, f)=\left(\begin{array}{c}
E^{x, x} \\
E^{x, y} \\
E^{x, z}
\end{array}\right)
$$

The Extended Nijboer-Zernike theory provides an analytical expression for the electric field vector:

$$
\begin{aligned}
\mathbf{E}^{x}(r, \phi, f)= & -i \gamma \mathrm{NA}^{2} \exp \left[\frac{-i f}{u_{0}}\right] \sum_{n, m} i^{m} \beta_{n m}^{x} \exp [i m \phi] \times \\
& \left(\begin{array}{c}
V_{n m, 0}+\frac{\mathrm{NA}^{2}}{2} V_{n m, 2} \exp [2 i \phi]+\frac{\mathrm{NA}^{2}}{2} V_{n m,-2} \exp [-2 i \phi] \\
-\frac{i \mathrm{NA}^{2}}{2} V_{n m, 2} \exp [2 i \phi]+\frac{i \mathrm{NA}^{2}}{2} V_{n m,-2} \exp [-2 i \phi] \\
-i \mathrm{NA} V_{n m, 1} \exp [i \phi]+i \mathrm{NA} V_{n m,-1} \exp [-i \phi]
\end{array}\right) .
\end{aligned}
$$

A similar expression exists for $y$-polarized illumination. We have introduced the $V_{n m, j}$ functions. These functions show a resemblance with the $V_{n m}(r, f)$-functions appearing in the scalar treatment of the diffraction problem as given in Ref. ${ }^{9,10}$ The appropriate Bessel-series expansion of the $V_{n m, j}$ function is not reproduced here, but can be found elsewhere. ${ }^{18}$ We note that in general, the aberration coefficients $\beta_{n m}^{x}$ and $\beta_{n m}^{y}$ are complex numbers and depend on the polarization state of the illuminator.

\subsection{Unpolarized Illumination}

For regular lithographic patterns, such as linear gratings, improved exposure latitudes are obtained when a polarized illuminator is used. However, for many other lithographic cells, such as logic-type of cells with a more "random-like" pattern, the gain in performance is marginal. In this paper, we therefore treat a more practical case of aberration retrieval using an unpolarized illumination of the pinhole. The advantage is a considerable simplification of the retrieval procedure, as the PSF maintains its rotational symmetry for an aberration-free lens. The intensity is found by:

$$
I(r, \phi, f) \propto E^{x} \cdot E^{x *}+E^{y} \cdot E^{y *} .
$$

The next step is to calculate the Fourier components with respect to the azimuthal component, just as we did in the scalar case. In addition, we restrict the analysis to the rotationally symmetrical components. The $0^{t h}$ Fourier component is defined as:

$$
\Psi^{0}(r, f)=\frac{1}{2 \pi} \int_{-\pi}^{\pi} I(r, \phi, f) \mathrm{d} \phi .
$$

In our restricted analysis, the rotationally symmetrical term is written as

$$
\Psi^{0}=\left(\beta_{0}^{0}\right)^{2} \Phi_{0}^{0}+2 \beta_{0}^{0} \sum_{n=1}^{n_{\max }} \operatorname{Re}\left(\beta_{2 n}^{0} \Phi_{2 n}^{0}\right) .
$$

with

$$
\Phi_{n}^{0}=\Psi_{n ; 0,0}^{0}+2 \mathrm{NA}^{2} \Psi_{n ; 1,1}^{0}+\mathrm{NA}^{4} \Psi_{n ; 2,2}^{0} \text { where } \Psi_{n, k, l}^{0}=V_{0, k}^{0 *}(r, f) V_{n, l}^{0}(r, f) .
$$

Equations (10) and (11) together resemble Eq.4 of the low-NA theory. Indeed, one can show that for NA $\rightarrow 0$, the Eqs. (10) - (11) and (4) give the same result.

Similar to the low-NA case, the aberration coefficients $\beta_{2 n}^{0}$ from Eq. 10 are found by a matching procedure between the observed and theoretical Fourier components. 

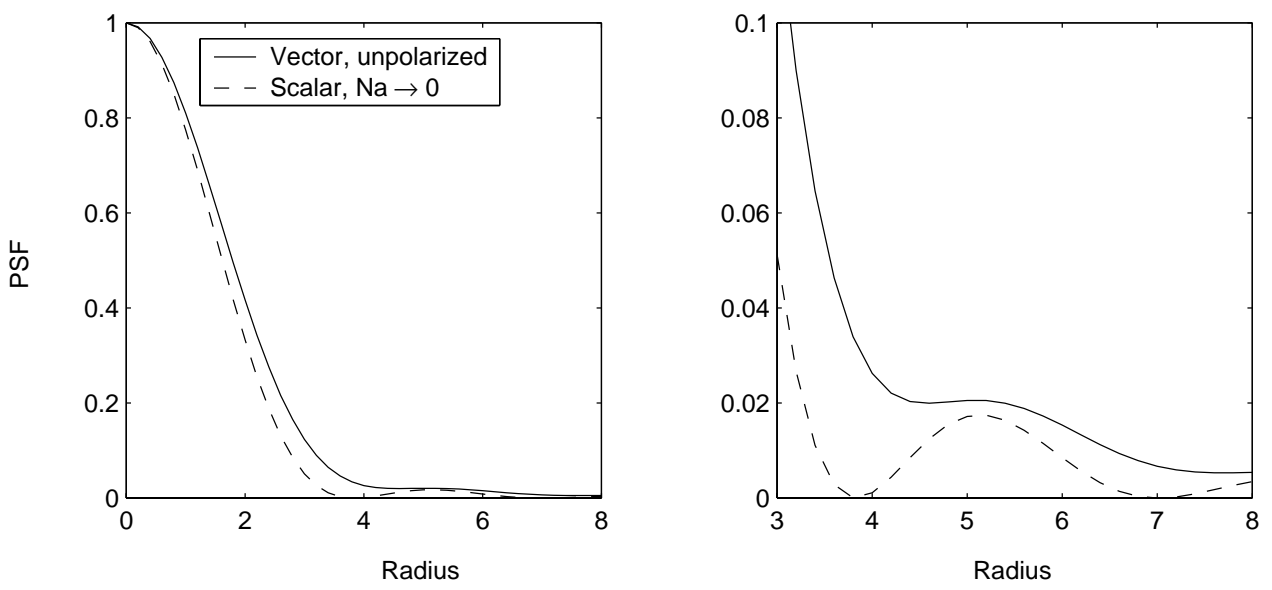

Figure 3. A comparison between a vectorial, unpolarized calculation (solid) and a scalar calculation (dashed) for a $N A=0.95$ system. The radial coordinate is dimensionless and properly scaled to $\lambda / \mathrm{NA}$. Left: the vectorial PSF is about $12 \%$ wider. Right: the enlargement shows that the vectorial PSF has no "zero", in contrast to the scalar PSF.

\subsection{Validating the high-NA formulas}

Figure 3 shows a comparison between a vectorial, unpolarized calculation and a scalar calculation for a NA = 0.95 system. Note that the vectorial calculation shows a PSF with a half-width that is somewhat larger compared to the scalar calculation. This result is important when we estimate diffusion constants. Figure 3 right shows an enlargement of the PSF, showing that the PSF in the vectorial case has no "zero", in contrast to the PSF in the scalar case.

We validate the high-NA formulas of the previous section by comparing the high-NA ENZ results to numerical simulations. $^{22}$ Figure 4 shows the PSF with the numerical aperture having the values NA $=0.60,0.85$ and 0.95 .
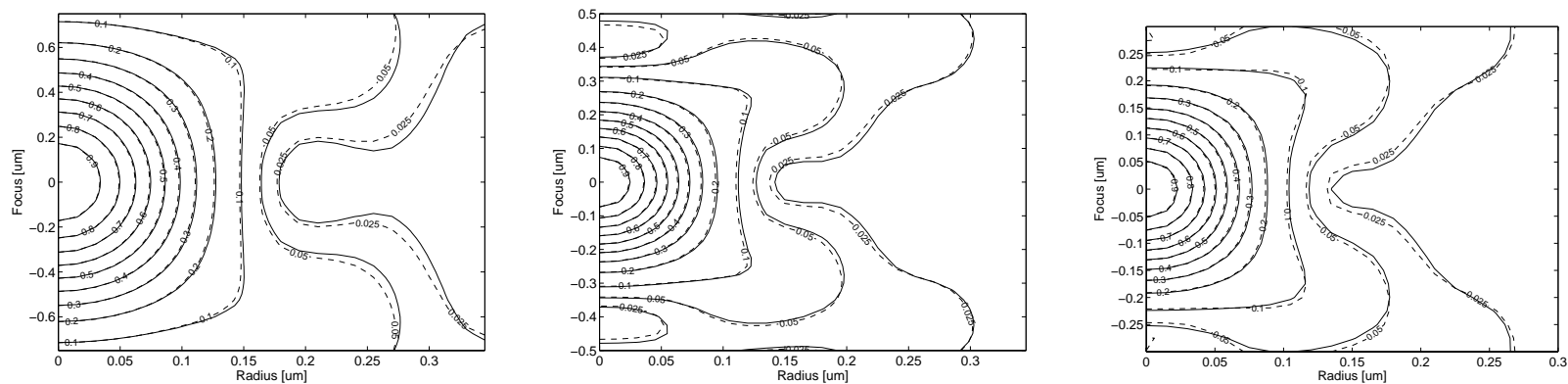

Figure 4. The point-spread function at various NA-values. The solid lines represent a simulation made with the lithographic simulator SOLID-C, the dashed lines represent the analytical high-NA ENZ calculation. Left: NA=0.60, mid: $N A=0.85$ and right: $N A=0.95$.

Up to now we have assumed that the diameter of the hole in the binary mask is so small that it can be regarded as a true delta function. From a practical point of view, however, it would be favourable to use holes with a non-negligible diameter since the increased amount of light allows a significant reduction of the required exposure dose, making the experimental procedure much more practical. We assume that the diameter is small compared to both the diffraction unit $\lambda / \mathrm{NA}$ in the object plane and the coherence radius of the illumination source, conditions that are almost always fulfilled. Here we take the same approach as for the scalar case. The effect of a non-negligible diameter is a drop in amplitude towards the rim of the pupil and can be effectively 
described by introducing a complex focus parameter, with optimal $d$ :

$$
f^{\prime}=f+i d \text {. }
$$

The optimal $d$ value is given in Ref. ${ }^{13}$ Figure 5 shows a calculation for a finite hole size, using Eq. 12 . Even
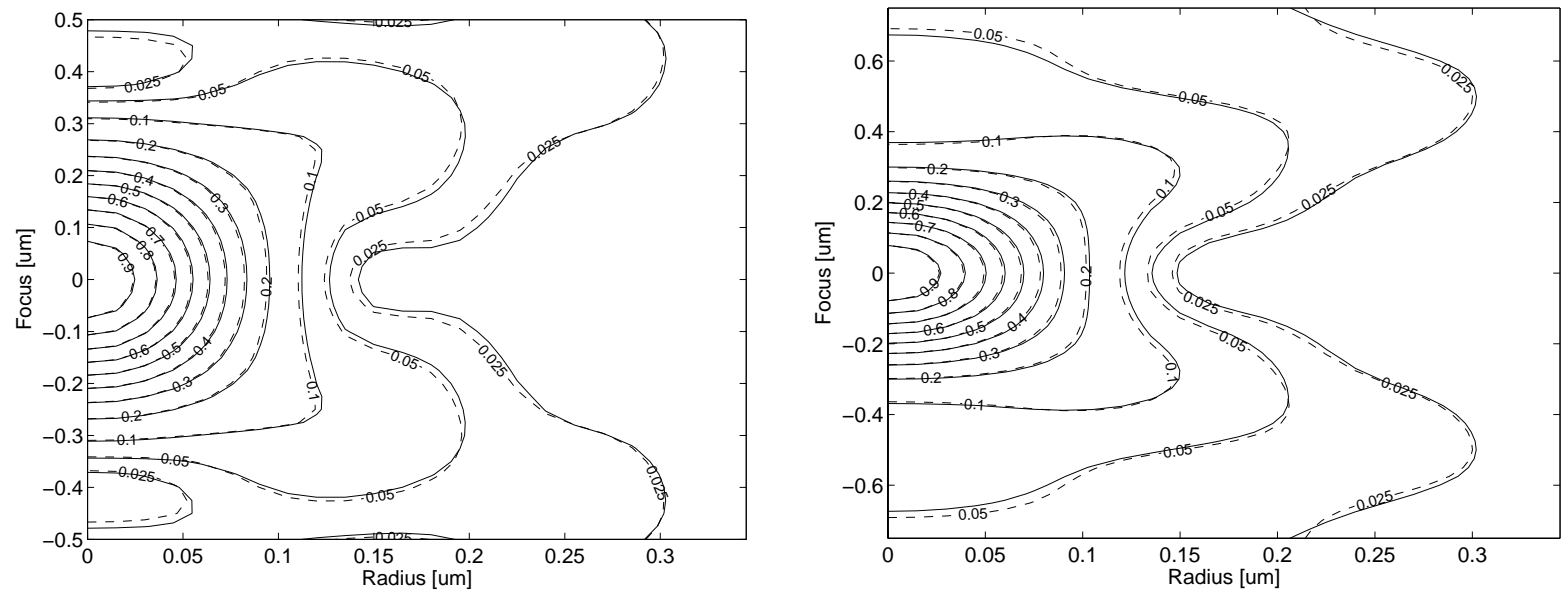

Figure 5. The point-spread function at two values of the hole diameter. The solid lines represent a simulation made with the lithographic simulator SOLID-C, the dashed lines represent the analytical high-NA ENZ calculation for an NA=0.85. Left: a diameter of $50 \mathrm{~nm}$. Right: a diameter of $200 \mathrm{~nm}$.

though the hole size of $200 \mathrm{~nm}$ is large for the $\mathrm{NA}=0.85$ system, a good agreement is obtained with the numerical results.

The effect of spherical aberration on the PSF can be calculated by adding an additional terms $\beta_{2 n}^{0}$ in Eq. 10 .
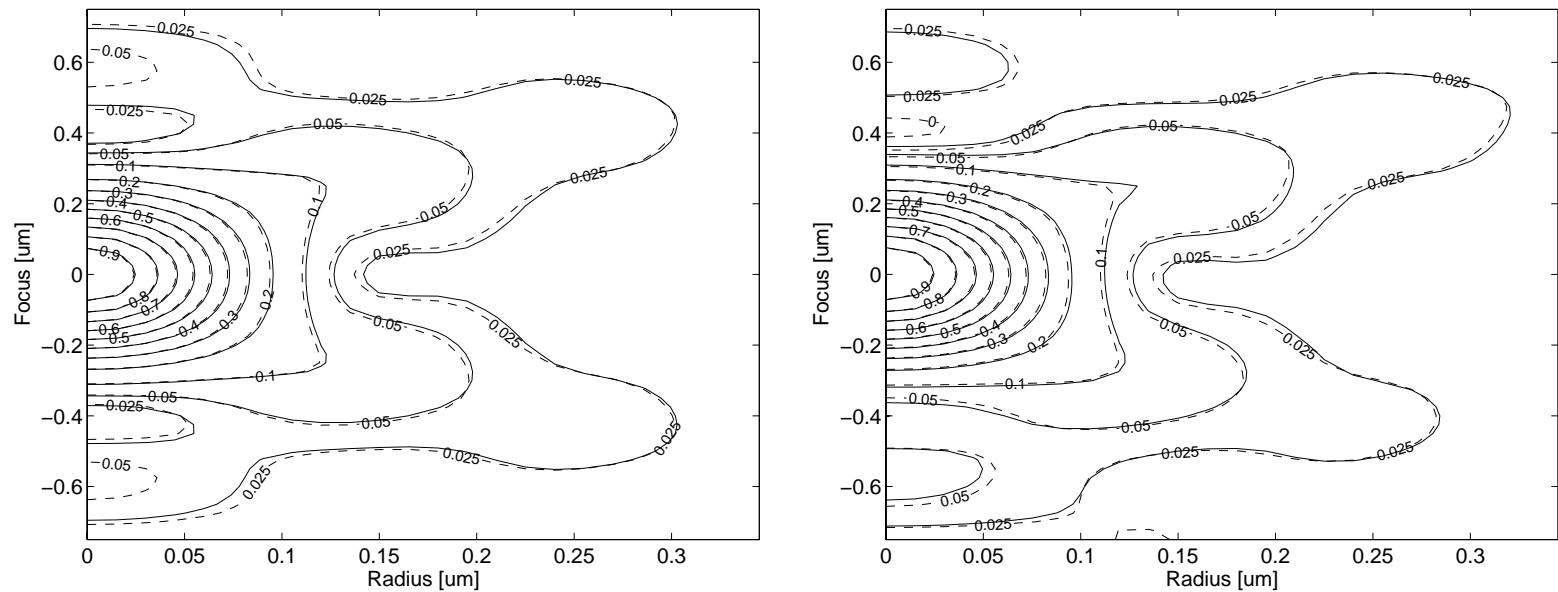

Figure 6. The point-spread function at two values of spherical aberration. The solid lines represent a simulation made with the lithographic simulator SOLID-C, the dashed lines represent the analytical high-NA ENZ calculation with NA=0.85. Left: aberration-free case. Right: $Z_{9}=40 \mathrm{~m} \lambda$ lowest order spherical aberration is added.

Figure 6 shows the effect of $Z_{9}=40 m \lambda$ lowest order spherical aberration. A through-focus asymmetry can be observed.

The case of liquid immersion lithography is easily calculated by scaling the numerical aperture and wavelength to the refractive index of the liquid, i.e. by replacing NA and $\lambda$ by NA/ $n_{\text {water }}$ and $\lambda / n_{w a t e r}$, respectively, throughout all formulas. Figure 7 compares the ENZ theory to numerical simulations, assuming water with a refractive 

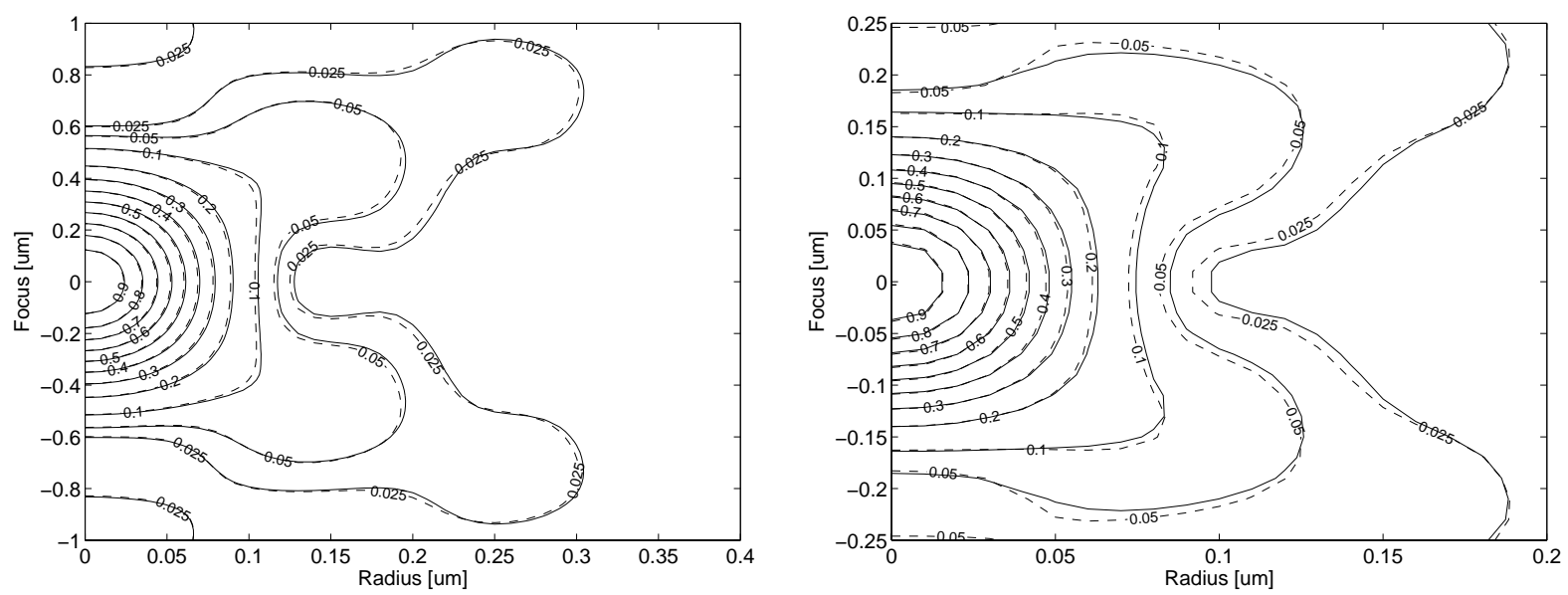

Figure 7. Two examples of the PSF for a water immersion system. The solid lines represent a simulation made with the lithographic simulator SOLID-C, the dashed lines represent the analytical high-NA ENZ calculation. Left: NA=0.85. Right: $N A=1.35$.

index $n_{\text {water }}=1.43664$. The calculations for a wet system with $\mathrm{NA}=0.85$ and $\mathrm{NA}=1.35$ are shown. Note the large depth of focus for the NA=0.85 case.

We take the same approach as in the low-NA case and adopt the DAIM model to account for image blur effects. There are a number of image blur effects: mechanical position noise, resist processing effects such as acid diffusion during the post exposure baking process, and a third cause for image blur originates from a stochastic variation of the focus parameter. To a good approximation, these effects can be taken into account by convolving the point-spread function in the radial variable $r$ and the focus variable $f$ by a Gaussian distribution function. It can be shown that the three effects, manifest themselves to a large extent additively in the ideal aberration-free point-spread function $\Phi_{0}^{0}$. Figure 8 shows an example of the PSF, where the diffusion length is 0 and $40 \mathrm{~nm}$,
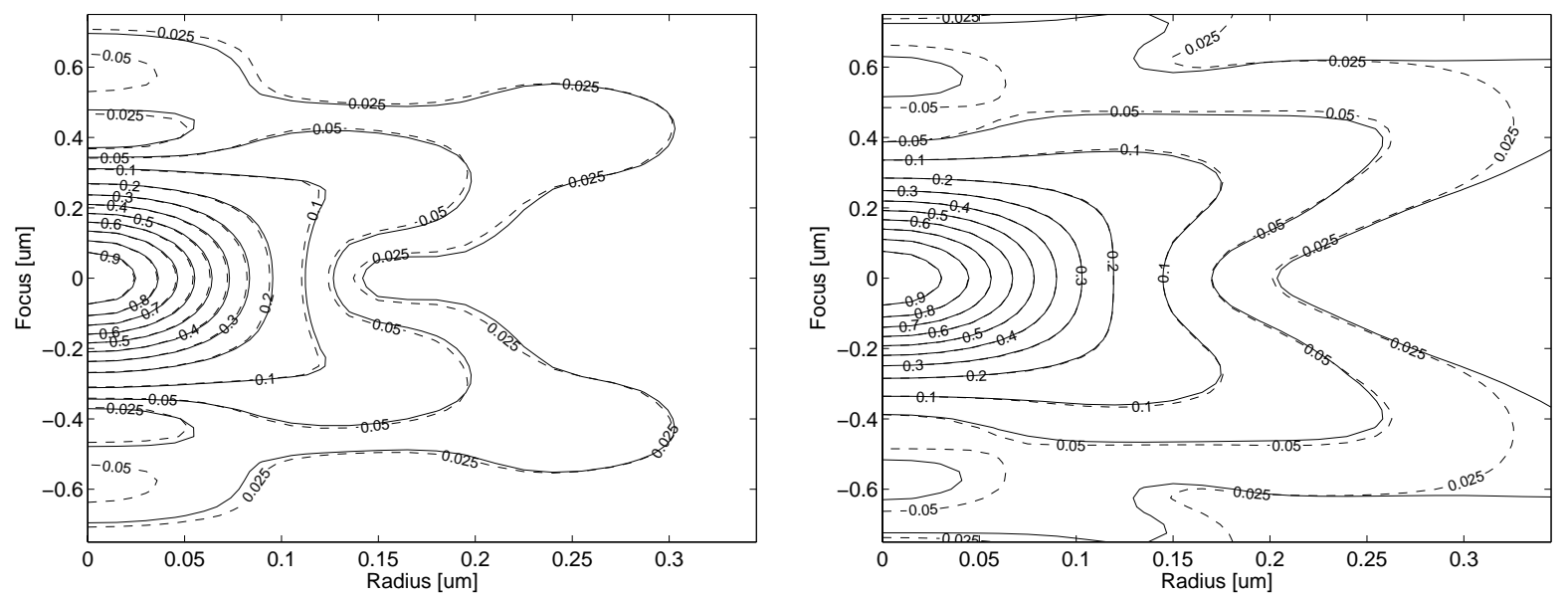

Figure 8. The impact of diffusion. The solid lines represent numerical simulations, dashed lines represent the high-NA ENZ calculations. Left: aberration-free PSF of an NA=0.85 system, no diffusion. Right: The same, but now with a diffusion length of $40 \mathrm{~nm}$.

respectively.

By comparing the ENZ values with the results obtained by numerical integration, it can be shown that the intrinsic accuracy of the ENZ theory is better then $10^{-6}$. The observed maximum absolute deviation of $1.5 \%$ 
between our analytical results and the numerical simulator ${ }^{22}$ is likely to be caused by the choice of the grid size, the representation of the finite hole and aliasing effects in the FFT procedure.

\section{HIGH-NA RETRIEVAL}

The above examples demonstrate that the high-NA ENZ calculations accurately describe the through-focus PSF. For unpolarized light, the retrieval scheme for aberrations and DAIM parameters is similar to the scalar approach and is also described in Section 2.1.

The experimental procedure to measure the PSF using SEM resist images is described in detail elsewhere. ${ }^{13}$ We use a simple chrome-on-quartz reticle with a, $0.6 \mu \mathrm{m}$ at reticle level, transparent hole. The reticle is exposed in a focus exposure matrix (FEM). A SEM, under job control, collects all images. The data reduction, from SEM image to through-focus intensity PSF, is done off-line. The exposures are made by two different scanners: 1) an ASML PAS5500/1150, a dry scanner system with $\lambda=193 \mathrm{~nm}, \mathrm{NA}=0.75$ projection lens, unpolarized illumination and 2) an ASML twinscan XT:1250i, immersion scanner system with $\lambda=193 \mathrm{~nm}, \mathrm{NA}=0.85$ projection lens, unpolarized illumination.

Figure 9 shows the an example of the pinhole experiment, where the exposures are made on the immersion $\mathrm{NA}=0.85$ scanner. The near perfect through-focus symmetry indicates a low level of spherical aberrations. For

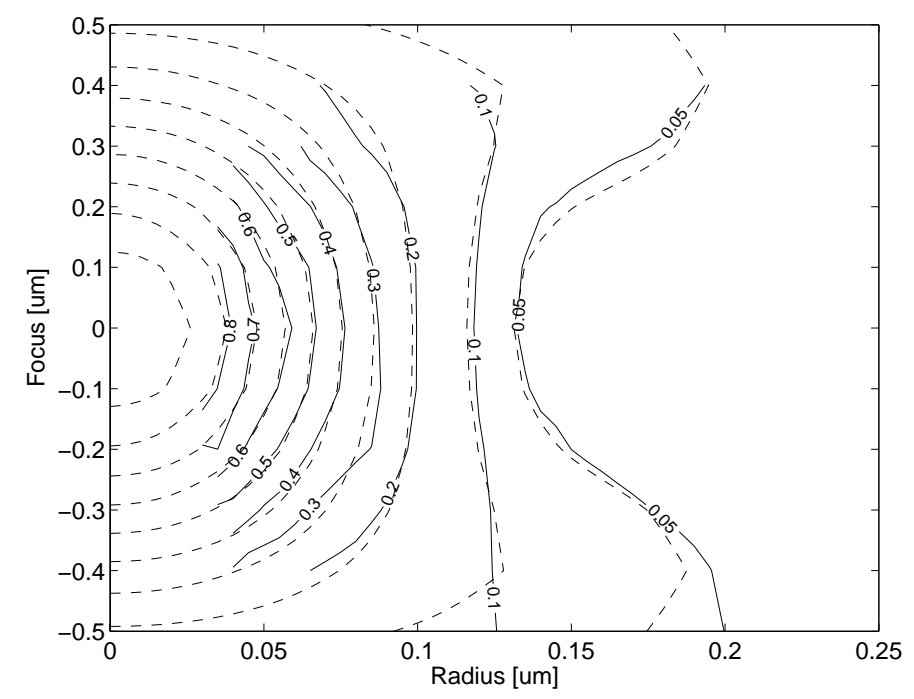

Figure 9. The experimental PSF (solid lines) and the PSF from the high-NA ENZ theory (dashed lines), where the exposures are made on the immersion $\mathrm{NA}=0.85$ scanner. The near perfect through-focus symmetry indicates a low level of spherical aberrations, below $10 \mathrm{~m} \lambda$. The photo resist is PAR-817. For the DAIM parameters we find: $\sigma_{r}=16, \sigma_{f}=$ $100 \mathrm{~nm}$.

the next experiment the exposures were made on the dry NA $=0.75$ scanner. We varied the post exposure baking (PEB) time in order to illustrate the increased diffusion length. Figure 10 shows two experimental examples of the pinhole experiment at two different PEB times. Note that extending the PEB time increases the diffusion parameter from $\sigma_{r}=26$ to $48 \mathrm{~nm}$. However, the focus noise parameter and aberration coefficients are the same, as expected.

Figure 11 shows the measured diffusion parameter $\sigma_{r}$ of the DAIM model versus the PEB time for GARS8105 resist (Fuji Film). We observe the expected increase of $\sigma_{r}$ in time, caused by acid diffusion. The data fit, indicated by the lines, represents a $\sqrt{D \cdot t+\text { offset }}$ function. The low-NA analysis, as we have also observed in previous experiments, ${ }^{15}$ shows an offset. In contrast, the high-NA analysis shows a nearly zero offset. The offset reduction is caused by the fact that the basic intensity functions we use for the new analysis are now calculated by the full vectorial ENZ theory and have a somewhat larger halfwidth compared to the basic intensity functions from the scalar theory, see Fig. 3 . 

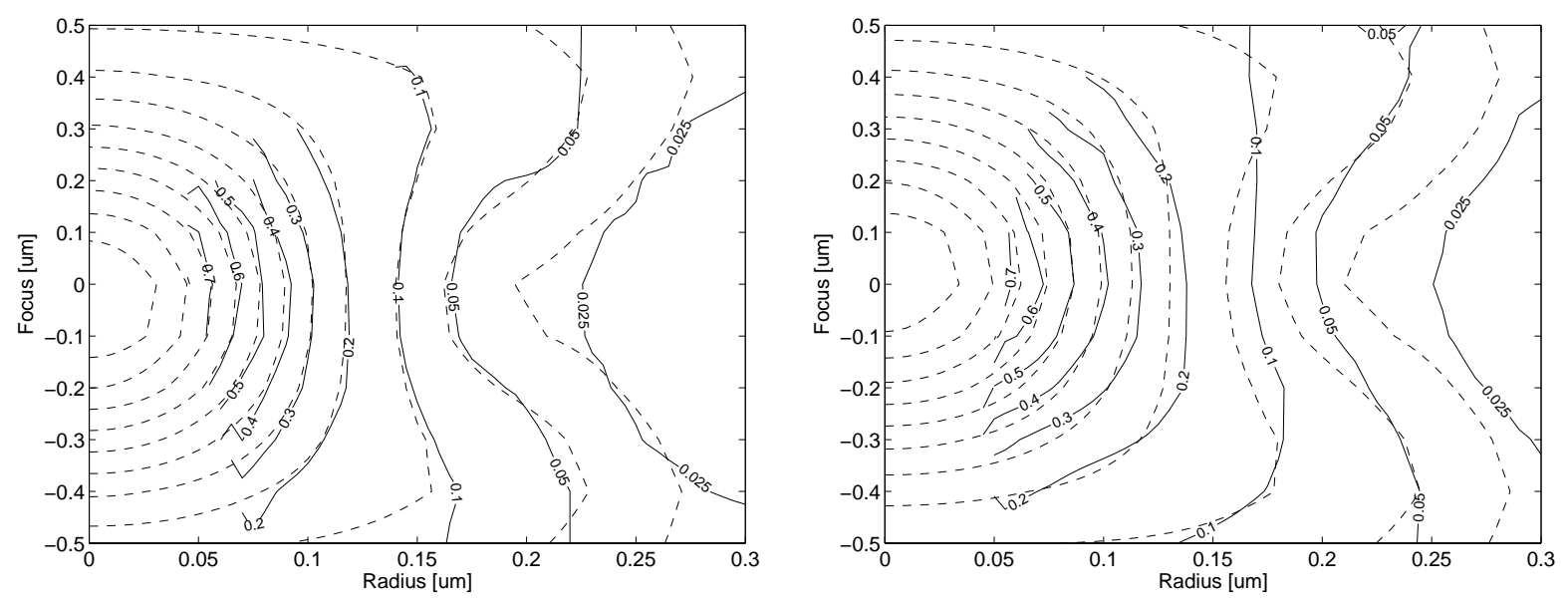

Figure 10. The experimental PSF (solid lines) and the PSF from the high-NA ENZ theory (dashed lines), where the exposures are made on the dry NA $=0.75$ scanner. The near perfect through-focus symmetry indicates a low level of spherical aberrations, below $10 \mathrm{m \lambda}$. The photo resist is GARS-8105. Left: the results for the standard PEB time of 90 seconds. For the DAIM parameters we find: $\sigma_{r}=26, \sigma_{f}=110 \mathrm{~nm}$. Right: The PEB time is extended to 270 seconds, we find $\sigma_{r}=48, \sigma_{f}=110 \mathrm{~nm}$

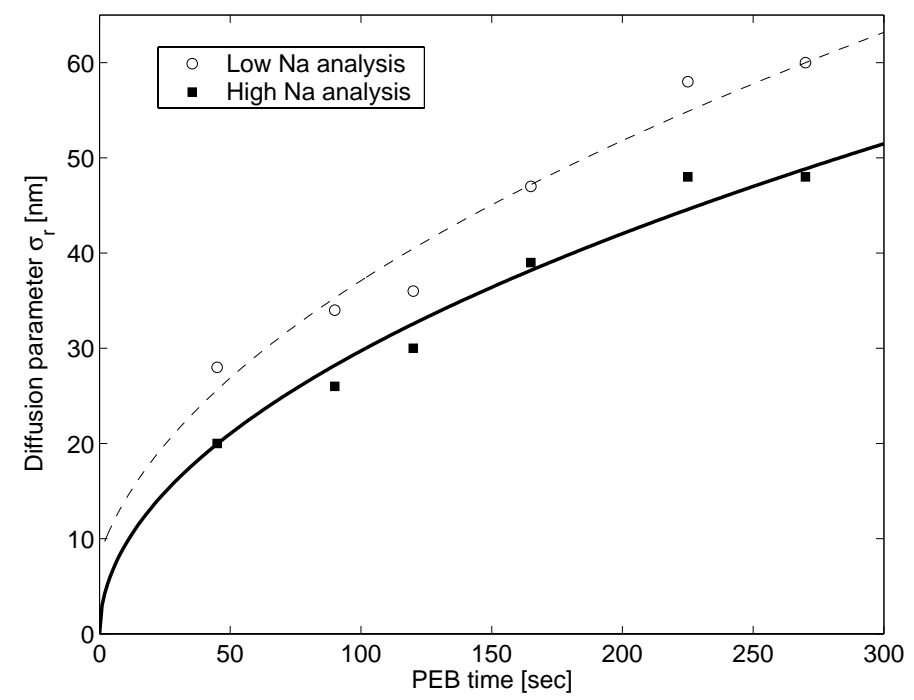

Figure 11. The measured diffusion parameter $\sigma_{r}$ versus the PEB time for the GARS-8105 resist. The open circles represents the $\sigma_{r}$ values obtained by the low-NA analysis. The closed square symbols represent the $\sigma_{r}$ values obtained by the high-NA analysis. The lines represent a data fit to a $\sqrt{D \cdot t+\text { offset }}$ diffusion law. Note that the fitted curve for the high-NA case (solid line) passes nearly through the origin.

\section{SUMMARY}

In this paper we have emphasized the high-NA extension of the Extended Nijboer-Zernike approach and we found a good agreement between theory, numerical simulations and experiment. For unpolarized light a retrieval scheme was presented that is similar to the low-NA case requiring modified basic intensity functions. In addition, we also accounted for various effects of image blur. The solution of the inverse problem, the evaluation of the Zernike coefficients and the diffusion and focus-noise parameters, is obtained by using the high-NA Extended Nijboer-Zernike approach. The method also applies to immersion systems with a very high NA. 


\section{ACKNOWLEDGMENTS}

Philips Research Leuven makes use of the cleanroom facilities of IMEC. The support from the IMEC litho department, as well as that of the IMEC P-Line is greatly appreciated. The authors wish to thank David Van Steenwinckel, Hans Kwinten and Mike Benndrof from Philips Research Leuven als well as David Laidler from IMEC for their helpfull discussions and experimental support.

\section{REFERENCES}

1. M. Born and E. Wolf, Principles of Optics (4th rev. ed., Pergamon Press, New York, 1970), sec. 8.8

2. T.A. Brunner, "Impact of lens aberrations on optical lithography", Proceedings of the Microlithography Seminar Interface, p. 1 (1996)

3. D.G. Flagello, H. van der Laan, J. van Schoot, I. Bouchoms, B. Geh, "Understanding systematic and random CD variations using predictive modelling techniques", Proc. SPIE 3679, p. 162 (1999)

4. C. Progler, D. Wheeler, "Optical lens specifications from the user's perspective", Proc. SPIE 3334, p.256 (1998)

5. D. G. Flagello, B. Geh, "Lithographic lens testing: analysis of measured aerial images, interferometric data and photo resist measurements", Proc. SPIE 2726, p.788 (1996)

6. D. Fuard, M. Besacier, P. Schiavone, "Assessement of different simplified resist models", Proc. SPIE 4691, p. 1266 (2002)

7. D. Fuard, M. Besacier, P. Schiavone, "Validity of the diffused aerial image model: an assessment based on multiple test cases", Proc. SPIE 5040, p. 1536 (2003)

8. B.R.A. Nijboer, Thesis, University of Groningen, 1942

9. A.J.E.M. Janssen, "Extended Nijboer-Zernike approach for the computation of optical point-spread functions", JOSA A 19, p. 849 (2002)

10. J. Braat, P. Dirksen, A.J.E.M. Janssen, "Assessment of an Extended Nijboer-Zernike approach for the computation of optical point-spread functions", JOSA A 19, p. 858 (2002)

11. P. Dirksen, J. Braat, P. De Bisschop, A.J.E.M. Janssen, Casper Juffermans and Alvina Williams, "Characterization of a projection lens using the Extended Nijboer-Zernike approach", Proc. SPIE 4691, p. 1391 (2002)

12. D. Van Steenwinckel, J. H. Lammers, L. H. A. Leunissen, and J. A. J. M. Kwinten, "Lithographic Importance of Acid Diffusion in Chemically Amplified Resists", Proc. SPIE, 5753, this conference, (2005)

13. P. Dirksen, J.J.M. Braat, A.J.E.M. Janssen, and C. Juffermans, "Aberration retrieval using the Extended Nijboer-Zernike approach", J. Microlith. Microfabr. Microsyst. 2, p. 61 (2003)

14. P. Dirksen, J. Braat, A. Janssen, C. Juffermans, A. Leeuwestein, "Experimental determination of lens aberrations from the intensity point-spread function in the focal region", Proc. SPIE 5040, p. 1 (2003)

15. P. Dirksen, J.J.M. Braat, A.J.E.M. Janssen, A. Leeuwestein, H. Kwinten, D. Van Steenwinckel, "Determination of resist parameters using the Extended Nijboer-Zernike theory", Proc. SPIE 5377, p. 13 (2004)

16. A.J.E.M. Janssen, J. Braat, P. Dirksen, "On the computation of the Nijboer-Zernike aberration integrals at arbitrary defocus", J. Mod. Opt., 20, p. 687 (2004)

17. C. van der Avoort, J.J.M. Braat, P. Dirksen, A.J.E.M. Janssen, "Aberration retrieval from the intensity point-spread function in the focal region using the Extended Nijboer-Zernike approach", submitted to J. Mod. Opt., 2005

18. J.J.M. Braat, P. Dirksen, A.J.E.M. Janssen, A. van der Nes, "Extended Nijboer-Zernike representation of the vector field in the focal region of an aberrated high-aperture optical system" , JOSA A, p. 2281 (2003)

19. J.J.M. Braat, P. Dirksen, A.J.E.M. Janssen, A.S. van de Nes, "Complex pupil function reconstruction at high numerical aperture using the Extended Nijboer-Zernike diffraction theory", Annual Meeting Optical Society of America, Rochester NY, 10-14 October 2004

20. J.J.M. Braat, P. Dirksen, A.J.E.M. Janssen A.S. van de Nes, "Aberration and birefringence retrieval in a high-aperture optical system using the Extended Nijboer-Zernike analysis", submitted to JOSA A, 2005

21. Additional information can be found on the Extended Nijboer-Zernike website: http://www.nijboerzernike.nl 
22. SOLID-C, a software product (release 6.4) of SIGMA-C GmbH, Thomas-Dehlerstrasze 9, D-81737 Munich, Germany. 